\title{
Etude sur modèle réduit du glissement de Léaz dans la retenue de Génissiat
}

\author{
J. Selmi et F. Fruchart \\ Compagnie Nationale du Rhône
}

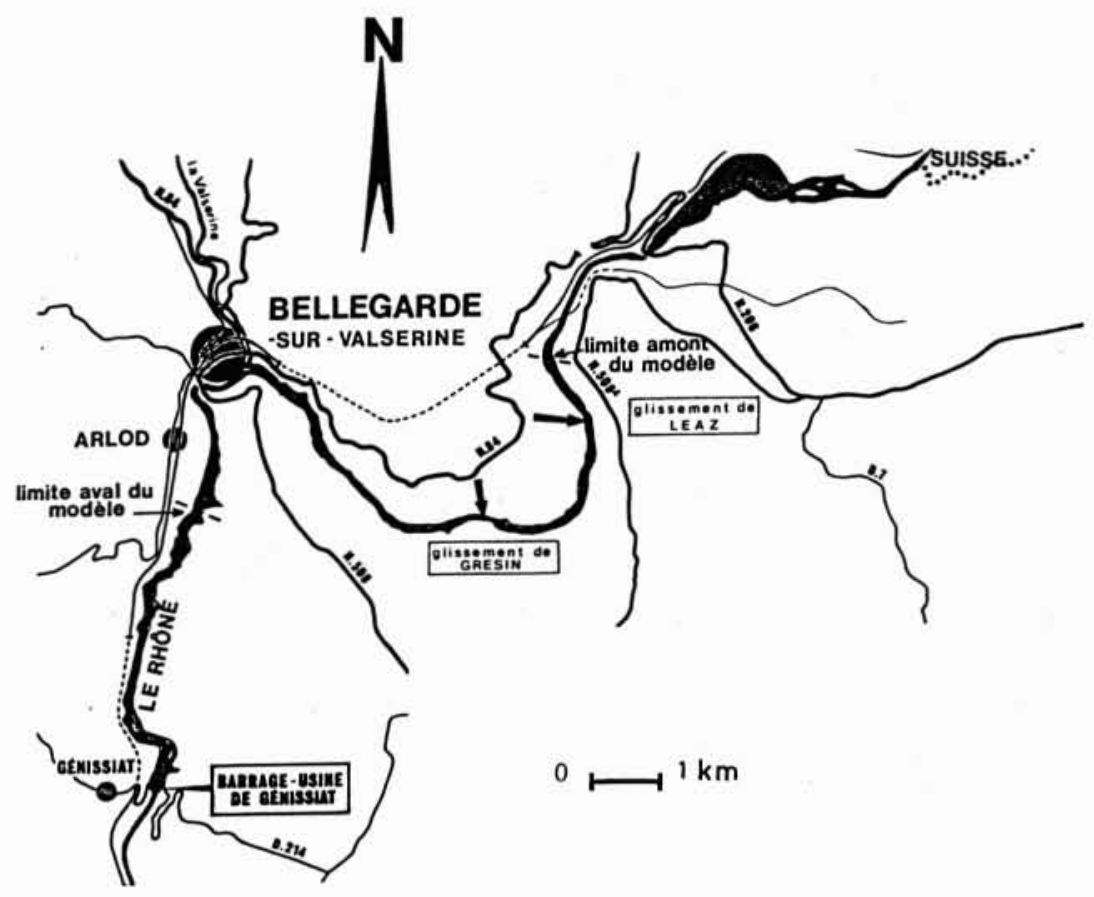

1. Plan de situation.

\section{Introduction}

Un glissement de terrain potentiel est situé en rive droite du Rhône, sur la commune de Léaz, au-dessus de la retenue créée par le barrage de Génissiat, distant de $25 \mathrm{~km}$ environ de la frontière suisse, comme le montre le plan de la figure 1 .

L'importance de ce glissement, dont le volume de la zone la plus active est estimé à $350000 \mathrm{~m}^{3}$, et la présence de l'agglomération de Bellegarde-sur-Valserine $7 \mathrm{~km}$ environ en aval, en bordure de la retenue, ont conduit la Compagnie Nationale du Rhône à y effectuer une surveillance étroite, et diverses études destinées à établir une consigne d'exploitation d'urgence, permettant d'éviter tous dommages pour les riverains, dus à l'onde provoquée par ce glissement.
Afin de préciser cette consigne, la Compagnie Nationale du Rhône a effectué une étude hydraulique précise sur les conséquences du glissement de Léaz dans la retenue de Génissiat, et en particulier pour la ville de Bellegardesur-Valserine.

Cette étude, basée sur un modèle réduit physique à l'échelle $1 / 200$, a été effectuée dans son Laboratoire d'hydraulique et d'essais de matériaux situé à Lyon.

Profitant de la réalisation de ce modèle, une étude complémentaire, concernant un autre glissement potentiel dont le volume est moindre, situé sur la commune de Grésin, en rive droite de la retenue, à $4,5 \mathrm{~km}$ en amont de Bellegarde-sur-Valserine (fig. 1), a également été effectuée.

Le déroulement et les principaux résultats de cette étude sont présentés ci-après.

(English Summary on page 71) 


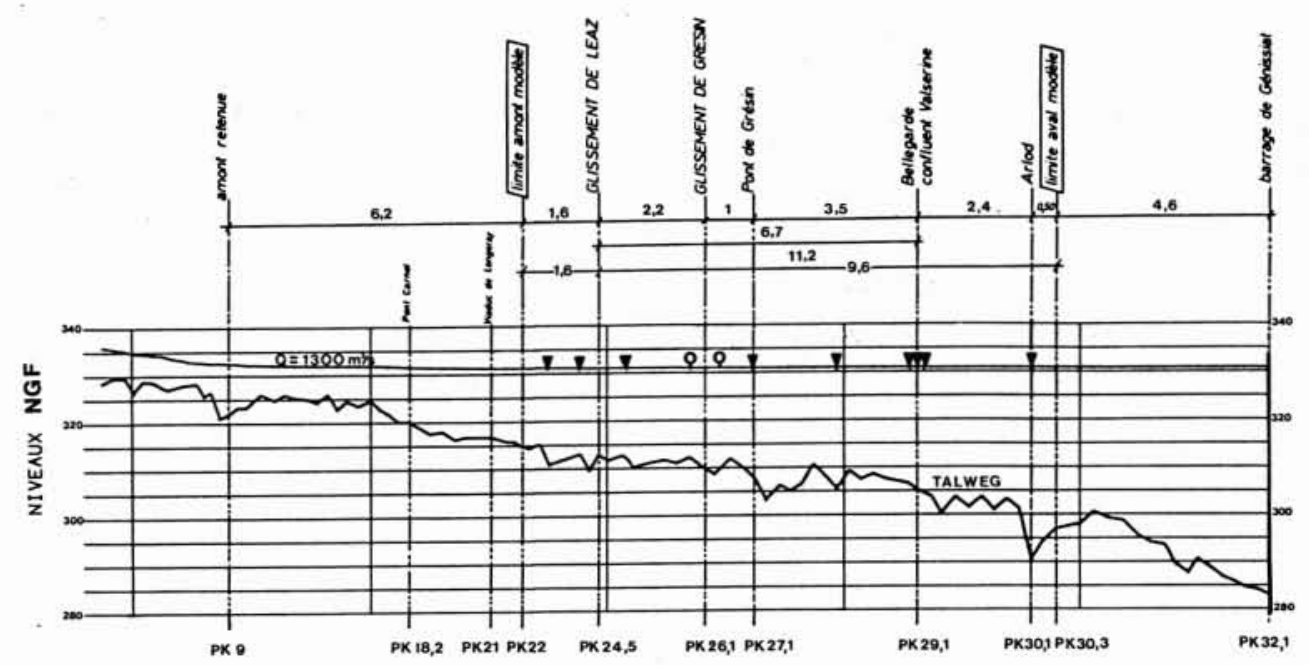

2. Profil en long de la retenue de Génissiat.

$\nabla$ Sondes limnimétriques pour étude du glissement de Léaz.

O Sondes limnimétriques supplémentaires pour étude du glissement de Grésin.

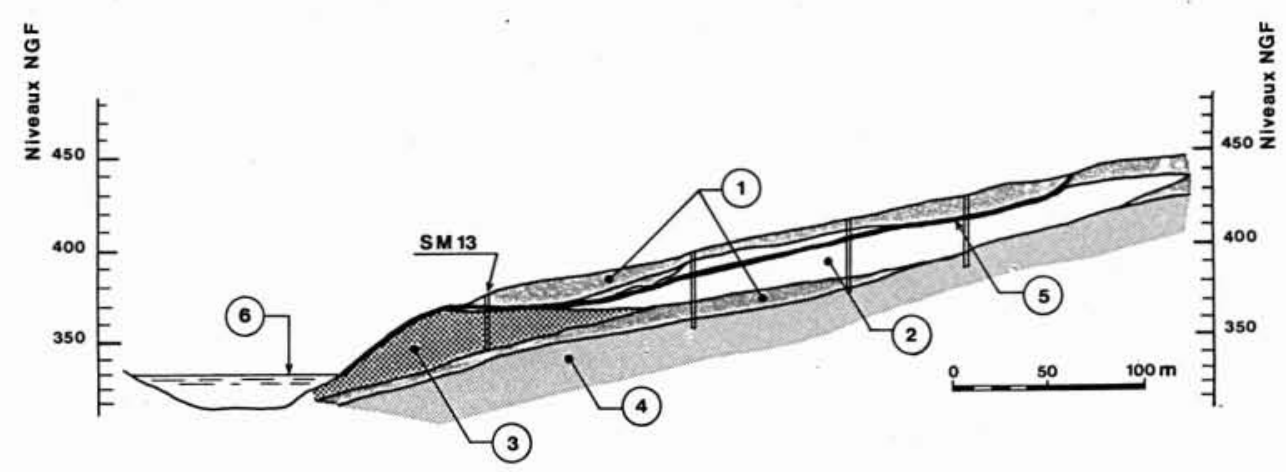

3. Coupe géologique du glissement de Léaz.
(1) Argile sableuse à galets et blocs.
(2) Argile grise silteuse varvée.
(3) Graviers, galets, sables (alluvions)
(4) Marnes argileuses et grès (substratum).
(5) Surface de glissement.
(6) Niveau de la retenue.

\section{But de l'étude}

L'étude doit déterminer dans une première phase, les conséquences du glissement de Léaz sur l'évolution des niveaux de la retenue, au droit des zones sensibles.

Elle doit donc représenter, aussi fidèlement que possible, la génération des ondes dans la zone du glissement, leur composition, leur propagation et leur évolution jusqu'au droit de ces zones.

Mais, du fait que les caractéristiques du glissement sont, de par leur nature, assez imprécises, l'étude doit non seule- ment déterminer les conséquences liées au glissement le plus probable, mais également la sensibilité des résultats à des variations plus ou moins importantes de ses caractéristiques permettant ainsi d'établir une consigne d'exploitation d'urgence de la retenue offrant le maximum de sécurité.

Enfin, l'étude devra porter sur les conséquences de la rupture du barrage en terre, qui pourrait se former dans la retenue au droit du glissement.

Dans une seconde phase elle doit déterminer également les conséquences du glissement de Grésin. 


\section{Données utilisées pour l'étude}

\subsection{Caractéristiques de la retenue}

La retenue de Génissiat s'étend sur une longueur de $23 \mathrm{~km}$; elle est relativement étroite ( $100 \mathrm{~m}$ en moyenne), avec des berges généralement assez raides sauf à l'amont en queue de retenue où elle s'évase largement, et localement dans la zone de la commune de Bellegardesur-Valserine et du village d'Arlod.

Dans la zone du glissement de Léaz, la largeur de la retenue est d'environ $100 \mathrm{~m}$ et la profondeur de $18 \mathrm{~m}$. Le volume de la retenue, au droit du glissement est de $470000 \mathrm{~m}^{3}$ pour le niveau normal $330,70 \mathrm{NGF}$, il dépasse donc celui du glissement normal $\left(350000 \mathrm{~m}^{3}\right)$. En revanche, si la retenue est abaissée au niveau minimum d'exploitation soit $325,00 \mathrm{NGF}$, le volume d'eau au droit du glissement est inférieur à celui du glissement.

Les débits caractéristiques du Rhône entrant dans la retenue de Génissiat sont les suivants :

$\begin{array}{lr}\text { - débit moyen } & 360 \mathrm{~m}^{3} / \mathrm{s} \\ \text { - crue décennale } & 1550 \mathrm{~m}^{3} / \mathrm{s} \\ \text { - crue centennale } & 2100 \mathrm{~m}^{3} / \mathrm{s}\end{array}$

Le niveau de la retenue est fonction de l'exploitation de l'usine de Génissiat et de l'hydraulicité, mais compte tenu des sections offertes aux écoulements, les lignes d'eau sont pratiquement horizontales entre Léaz et le barrage, quel que soit le débit du Rhône, comme le montre la figure 2 .

En cas de glissement de terrain l'onde qui se déplacera vers l'amont n'entraînera pas de risques importants du fait de la topographie des lieux et de l'absence d'habitations, par contre, l'onde qui se déplacera vers l'aval, rencontrera des zones basses et habitées, situées au droit de la commune de Bellegarde, dans la zone du confluent de la Valserine, et du village d'Arlod.

En effet, au confluent de la Valserine, des habitations sont situées peu au-dessus du plan d'eau normal, et l'examen des fonds montre qu'il existe un cañon profond dans l'axe du Rhône et une plage submergée située devant les habitations, qui peut favoriser le déferlement.

A l'aval immédiat du confluent de la Valserine, des habitations, certes plus élevées, sont situées derrière un mur quasi perpendiculaire à l'axe de la retenue amont. On peut donc craindre dans ce secteur, une aggravation de l'amplitude de l'onde due à un effet de réflexion sur ce mur et de force centrifuge créée par le virage suivant.

Le village d'Arlod est situé près du plan d'eau mais la retenue y est assez large, et le tronçon amont qui le sépare de la Valserine, très sinueux et étroit, aura un effet amortisseur certain.

Le barrage de Génissiat est situé à $14 \mathrm{~km}$ du glissement. C'est un barrage poids ayant une revanche de plus de $5 \mathrm{~m}$ par rapport à la retenue normale. L'onde ne devrait pas être dangereuse pour cet ouvrage.

\subsection{Caractéristiques du glissement de Léaz}

Ce glissement a fait l'objet de nombreuses études dont certaines ont été confiées à l'Association pour le dévelop- pement de la recherche des glissements de terrains (ADRGT).

La coupe géologique du glissement, perpendiculairement à l'axe de la retenue (fig. 3), montre qu'il est situé sur une pente faiblement inclinée $\left(10^{\circ}\right)$ nettement au-dessus de la retenue $(+35 \mathrm{~m})$.

Il a été bien établi que le niveau de la retenue n'a aucune influence sur la dynamique du glissement, et la consigne de sécurité consistant à abaisser rapidement le plan d'eau en cas de signes précurseurs du glissement peut être effectuée sans danger.

Sur la vue panoramique du glissement, objet de la figure 4, la zone instable correspond sensiblement à la partie boisée, située au-dessus de la rupture de pente. La partie inférieure, constituée de graviers crus, est stable.

Ce glissement est surveillé par divers dispositifs.

Le volume total de la zone du glissement est de $1400000 \mathrm{~m}^{3}$, mais le volume susceptible d'être mis en mouvement est de $350000 \mathrm{~m}^{3}$ environ. Ce glissement a comme caractéristiques géométriques :

$$
\begin{array}{lr}
\text { - longueur } & 350 \mathrm{~m} \\
\text { - largeur moyenne } & 100 \mathrm{~m} \\
\text { - épaisseur } & 10 \mathrm{~m}
\end{array}
$$

Les études effectuées montrent que ce glissement est de type viscoplastique et que la vitesse d'arrivée dans la retenue serait de $26 \mathrm{~m} / \mathrm{s}$.

\subsection{Le modèle réduit}

\subsubsection{Modélisation de la retenue}

La génération de l'onde qui a été estimée impossible à déterminer par modèle mathématique, devait donc être étudiée à minima sur un modèle physique limité à la zone de glissement.

La propagation de l'onde en revanche, aurait pu être calculée à partir des résultats obtenus aux limites de ce modèle physique, mais le caractère violent de l'onde initiale attendue (onde à front raide), sa complexité liée aux effets de réflexion et d'amortissement des différents virages et talwegs affluents, la topographie particulière de la retenue au droit de Bellegarde, ont conduit à opter pour un modèle réduit couvrant le tronçon de retenue compris entre le glissement de Léaz et les zones sensibles. Ce modèle physique présente également l'avantage d'être plus démonstratif pour les riverains.

Le modèle couvre donc $11,2 \mathrm{~km}$ de retenue depuis l'amont du glissement de Léaz jusqu'à l'aval du village d'Arlod.

L'échelle choisie étant $1 / 200$, le modèle a $56 \mathrm{~m}$ de longueur.

Le modèle est exploité en respectant la similitude de Froude, qui consiste à prendre comme invariant dans la nature et le modèle, le nombre sans dimension :

$$
F r=\frac{V}{\sqrt{g h}} \text { où } \begin{aligned}
& g \cdot \text { vitesse } \\
& h \text { accélération de la pesanteur } \\
& \text { hauteur d'eau }
\end{aligned}
$$


4. Vue aérienne de la zone du glissement de Léaz, depuis la rive gauche.

La zone du glissement correspond à la zone boisée située en rive droite de la retenue, avant le virage que fait le Rhône.

Noter:- l'étroitesse de la retenue, - la ville de Bellegarde-sur-Valserine en arrière-plan.

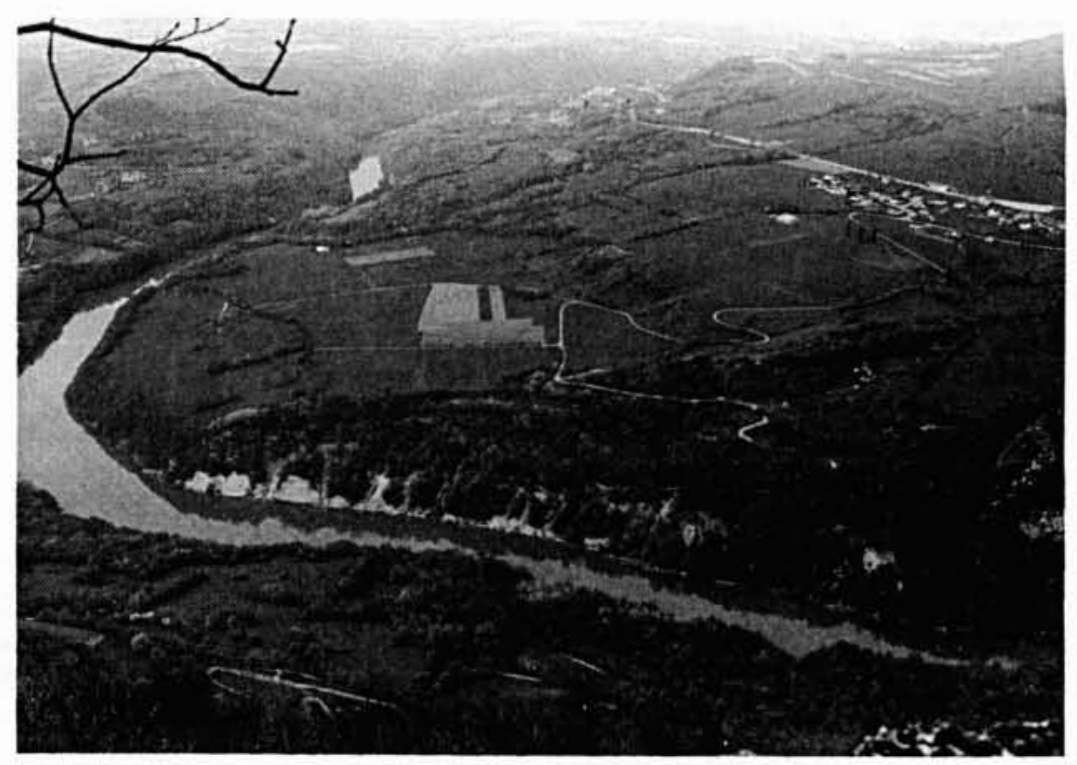

5. Vue depuis l'amont de l'ensemble du modèle réduit.

Au premier plan : l'amont du modèle et la zone du glissement.

Au fond: la partie aval du modèle avec la zone de Bellegarde.

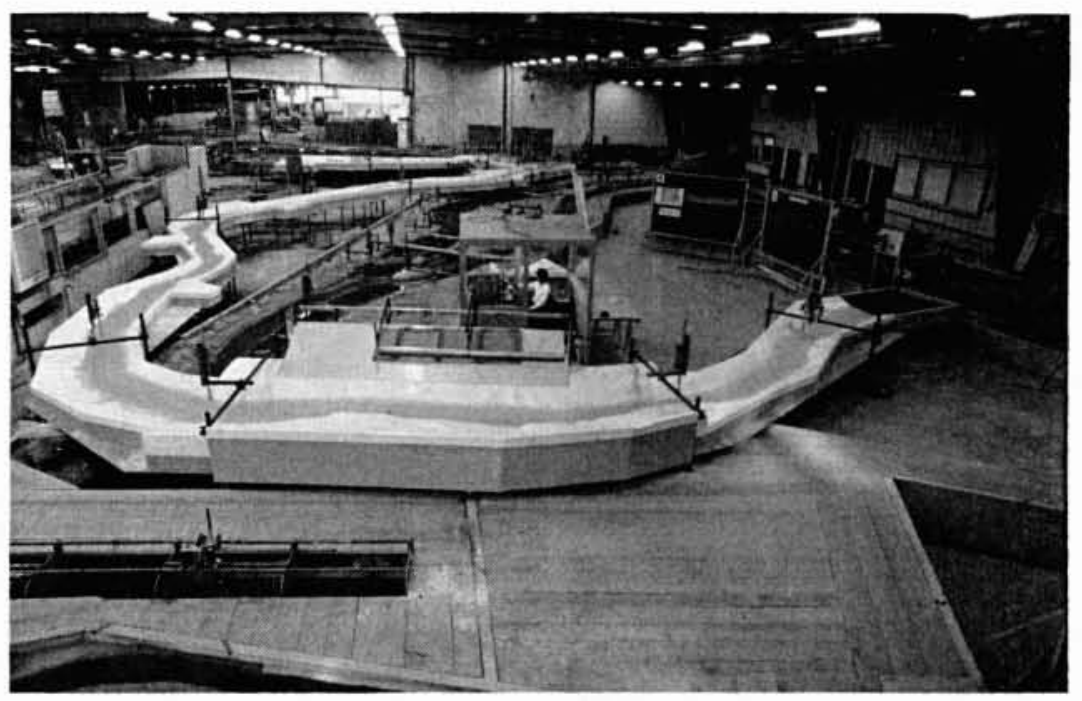

6. Vue depuis l'aval de l'ensemble du modèle réduit.

Au premier plan: Bellegarde-sur-Valserine. A droite: le village d'Arlod.

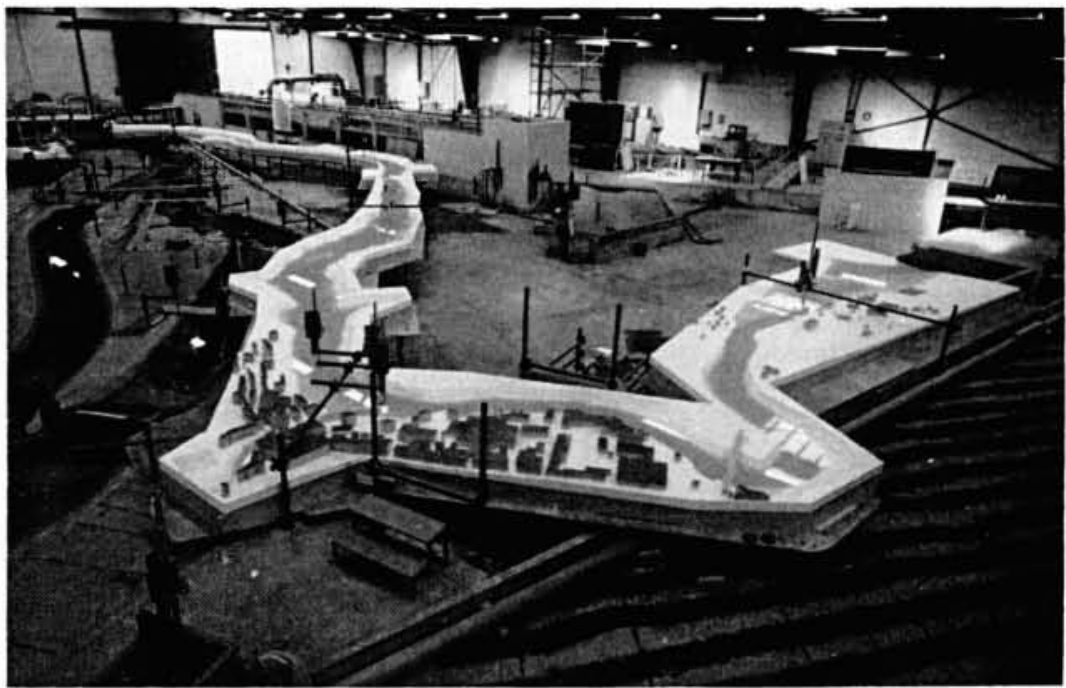




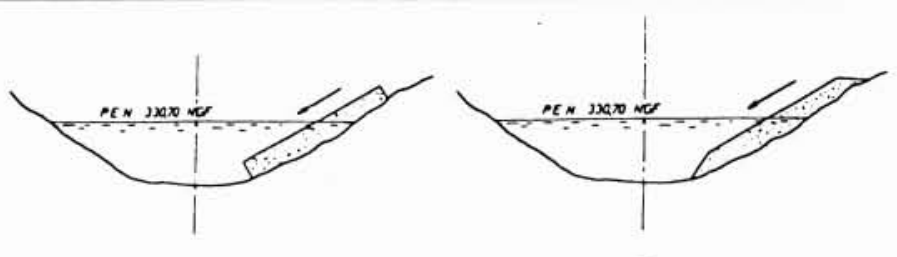

a)

A

B

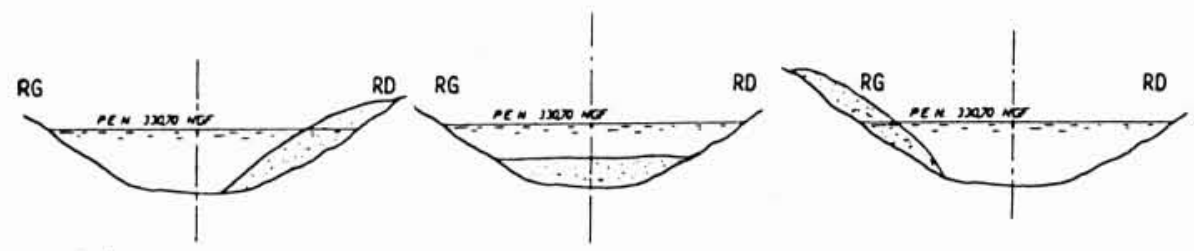

b)

7. Modélisation du glissement de Léaz.

a - formes du glissement,

b - positions finales du glissement.

Il doit être clair que le modèle réalisé est un modèle hydraulique et non rhéologique.

En particulier ne peuvent être reproduits strictement en similitude, les phénomènes de déformations éventuelles du glissement pendant la descente et au contact avec l'eau. C'est pourquoi le glissement a été représenté par différents matériaux, ayant des comportements mécaniques caractéristiques.

La topographie de la retenue a été modélisée précisément à partir des courbes de niveaux. Le modèle a été construit en polystyrẻne expansé, recouvert de résine polyester. La zone du glissement, relativement rectiligne, a été linéarisée sur le modèle, et remplacée dans la partie haute, par une plaque d'inclinaison réglable sur laquelle était disposé le glissement.

Une vue d'ensemble du modèle est présentée sur les figures 5 et 6 .

Le modèle comporte un dispositif d'alimentation en eau à l'amont, un volet de réglage du niveau aval et 9 sondes limnimétriques résistives, type détecteur de houle, destinées à mesurer l'évolution des niveaux au passage de l'onde, soit une sonde à l'amont du modèle, une sonde à l'amont et à l'aval immédiat du glissement, 2 sondes intermédiaires entre le glissement et Bellegarde, 3 sondes dans la zone sensible de Bellegarde et 1 sonde à Arlod (fig. 2).

Le coefficient de Strickler de la retenue modélisée, correspond à une rugosité réelle de $\mathrm{K}=38$ valeur conforme à celle de la retenue.

La végétation des berges et des terrains face au glissement, n'est pas représentée sur le modèle car d'une part elle est peu importante dans la nature, et d'autre part ceci va dans le sens de la sécurité.

\subsubsection{Modélisation du glissement}

Toute chose égale par ailleurs, l'onde dépend de la forme du glissement, et plus particulièrement du front du glis- sement au moment où celui-ci entre dans la retenue, et des deux situations présentées figure $7 a$ l'hypothèse B est certainement la plus probable.

Par ailleurs, des trois hypothèses de position finale du glissement, représentées sur la figure $7 b$, l'hypothèse centrale est la plus probable, et en tous les cas c'est elle qui déplacera le maximum de volume d'eau. Elle sera donc retenue pour les essais.

Le matériau utilisé pour les essais devra donc respecter les conditions ci-dessus.

Des matériaux pulvérulents pourraient, a priori, répondre assez bien à ces contraintes. Cependant, on peut se demander si l'effet mécanique est correctement reproduit du fait des vides. Les résultats pourraient être alors sousestimés.

Il est par ailleurs prudent de ne pas exclure un glissement de type monolithique ne se déformant pas dans la descente, qui donnerait un effet de choc maximum.

Il a donc été retenu d'effectuer les essais en considérant les deux cas suivants :

a) Glissement monolithique: Il est simulé par des blocs représentant $100000 \mathrm{~m}^{3}$ chacun, constitués en rhodorsil, qui est une résine silicone souple. Ces blocs épousent la forme du terrain lors de la descente, et s'adaptent à la forme du lit en position finale.

Notons que pour le glissement de Léaz, la chance veut que la forme du glissement initial soit assez voisine de la forme de la retenue qui sera occupée par le glissement, ce qui facilite sa simulation. Ceci n'est pas le cas pour le glissement de Grésin dont la largeur est beaucoup plus importante que la largeur de la retenue au droit du glissement. Ce matériau d'utilisation aisée a permis d'effectuer de nombreux essais destinés à étudier l'influence des différents paramètres.

Ce type de glissement a également été simulé par des sacs en plastique remplis de sable et d'eau, de volume unitaire 
équivalent à $25000 \mathrm{~m}^{3}$. Ce type de simulation correspond à un glissement peu déformable avec un front raide qui a permis d'étudier l'influence de la forme du front, du volume du glissement $\left(>350000 \mathrm{~m}^{3}\right)$ et de la densité.

b) Glissement avec déformation : Il est simulé par des matériaux tels que, graviers, galets de tailles différentes et sable.

Le dispositif d'essai est constitué d'une boîte représentant un volume de $350000 \mathrm{~m}^{3}$ comportant une trappe placée en biseau dans la partie inférieure, pour effectuer le lâcher.

\subsubsection{Mesure de la vitesse du glissement}

Les blocs simulant un glissement monolithique sont reliés par un fil à un potentiomètre rotatif mesurant le déplacement en fonction du temps, dont on déduit la vitesse du glissement.

Dans le cas de matériaux pulvérulents, le fil est relié à un élément noyé dans la masse du glissement, donnant une vitesse moyenne approximative.

Des chronophotographies sont également effectuées pendant les essais pour analyses de la formation des ondes et du déplacement.

\section{Essais et résultats}

\subsection{Méthodologie}

Le glissement de Léaz est, dans un premier temps, testé avec des caractéristiques égales à celles définies par l'ADRGT, et dans des conditions hydrologiques déterminées.

Cet essai qualifié « essai de base » a pour paramètres :

$\begin{array}{lc}\text { - volume } & 350000 \mathrm{~m}^{3} \\ \text { - vitesse } & 26 \mathrm{~m} / \mathrm{s} \\ \text { - densité } & 1,8 \\ \text { - débit du Rhône } & 0 \\ \text { - niveau de la retenue } & 330,70 \mathrm{NGF}\end{array}$

L'influence des divers paramètres est ensuite étudiée à partir de cet essai de base.

\subsection{Essai de base}

Le glissement représenté par des blocs de rhodorsil pénétrant dans la retenue, génère deux trains d'ondes se déplaçant, l'un vers l'amont, l'autre vers l'aval. La figure 8 schématise la génération de ces ondes et la figure 9 montre une vue du modèle en cours d'essais.

Le premier pic est dû à l'eau chassée latéralement par les extrémités du glissement et sa propagation est à front raide sur $400 \mathrm{~m}$ environ.

Lorsque le glissement se trouve au fond de la retenue, de l'eau le recouvre encore mais une partie de celle-ci commence à remonter la rive opposée.

A son apogée, cette masse d'eau recouvre entièrement cette rive jusqu'à $370,00 \mathrm{NGF}$ en moyenne, c'est-à-dire

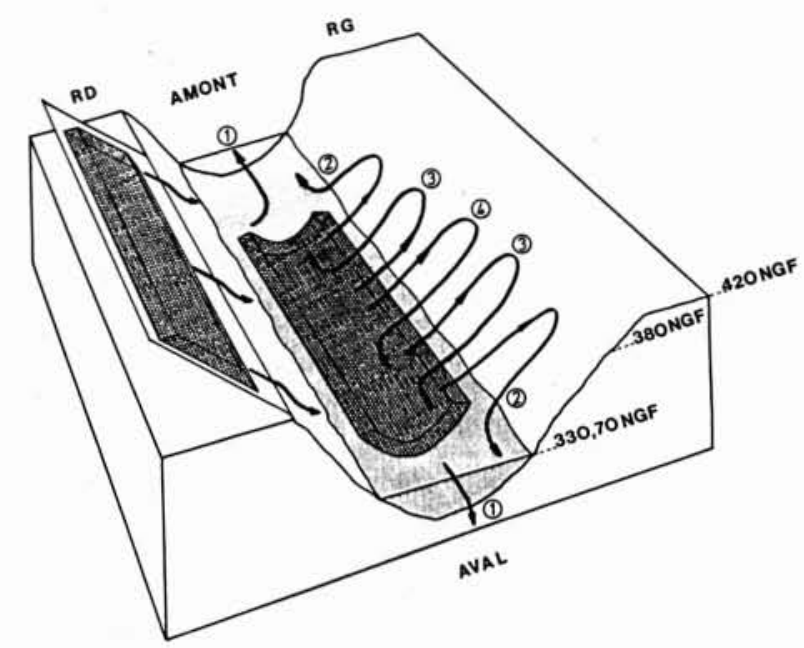

8. Glissement de Léaz - Essai de base.

Schéma de principe de la génération des trains d'ondes avec indication de l'origine des pics successifs.

$40 \mathrm{~m}$ environ au-dessus du plan d'eau normal, et localement $380,00 \mathrm{NGF}$.

L'eau projetée sur la rive gauche retombe ensuite dans la retenue et génère deux nouvelles ondes aux extrémités du remblai immergé comportant plusieurs pics (3 à l'aval, 2 à l'amont).

Les trains d'ondes se déplaçant vers l'amont et vers l'aval s'amortissent rapidement du fait des balancements et des réflexions dans les virages, et se regroupent en onde unique assez rapidement.

L'amplitude est :

de $4,80 \mathrm{~m}$ à proximité amont du glissement (pointe $\mathrm{n}^{\circ} 2$ premier pic)

de $3,40 \mathrm{~m}$ à proximité aval du glissement (pointe $\mathrm{n}^{\circ} 3$ deuxième pic)

de $1,60 \mathrm{~m}$ au pont de Grésin à 2,5 $\mathrm{km}$ du glissement (pointe $\left.\mathrm{n}^{\circ} 4\right)$

de $1,00,1,20$ et $1,40 \mathrm{~m}$ à Bellegarde-sur-Valserine (pointes $\mathrm{n}^{\text {os }} 6,7$ et 8 ) où l'onde est soumise aux effets de forces centrifuges et de réflexion)

de $0,5 \mathrm{~m}$ à Arlod.

Le temps de propagation de l'onde est de $13 \mathrm{~m} / \mathrm{s}$, il est du même ordre que celui tiré de la formule classique suivante :

$$
c=\sqrt{\frac{g s}{b}} \text { où } \begin{array}{lll}
c & \text { vitesse de l'onde } \\
& & \text { surface du profil en travers } \\
b & \text { largeur au miroir }
\end{array}
$$

La figure 10 présente l'onde mesurée en différents points du modèle. 


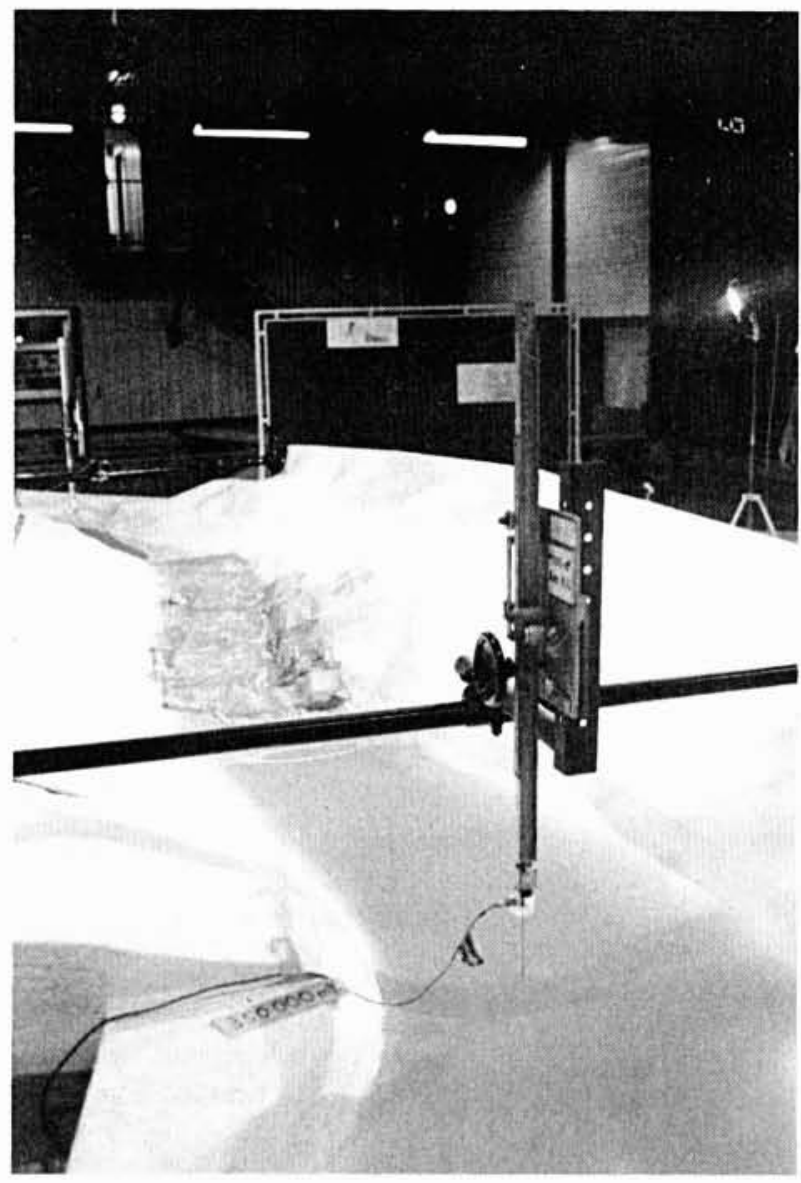

9. Glissement de Léaz — Vue du modèle en cours d'essais. Noter la formation du premier pic du train d'ondes aval et la submersion de la rive gauche entraînant la formation des pics suivants.

\subsection{Etude de l'influence de divers paramètres}

\subsubsection{Influence de la modélisation du glissement}

Les essais ont été effectués dans le cas du glissement de $350000 \mathrm{~m}^{3}$ monolithique, simulé successivement par des blocs de rhodorsil et des sacs de sable, et dans le cas du glissement déformable, avec des matériaux pulvérulents.

Les essais effectués avec des sacs de sable donnent un effet de choc plus important, dû à la forme plus raide du front du glissement. La masse d'eau projetée sur la rive opposée remonte à une altitude supérieure de $20 \mathrm{~m}$ par rapport à l'essai de base. Ce comportement différent génère un premier pic plus faible et des pics suivants plus forts. Après regroupement, l'onde résultante est à peine supérieure à celle de l'essai de base $(+0,10 \mathrm{~m})$.

Les essais effectués avec des matériaux pulvérulents, montrent que l'effet de choc est notablement réduit, et l'amplitude est toujours plus faible qu'avec le glissement monolithique. A Bellegarde, l'amplitude maximum n'est que de 1,0 $\mathrm{m}$ avec des gros galets, 1,2 $\mathrm{m}$ avec des petits galets, $1,3 \mathrm{~m}$ avec du sable.

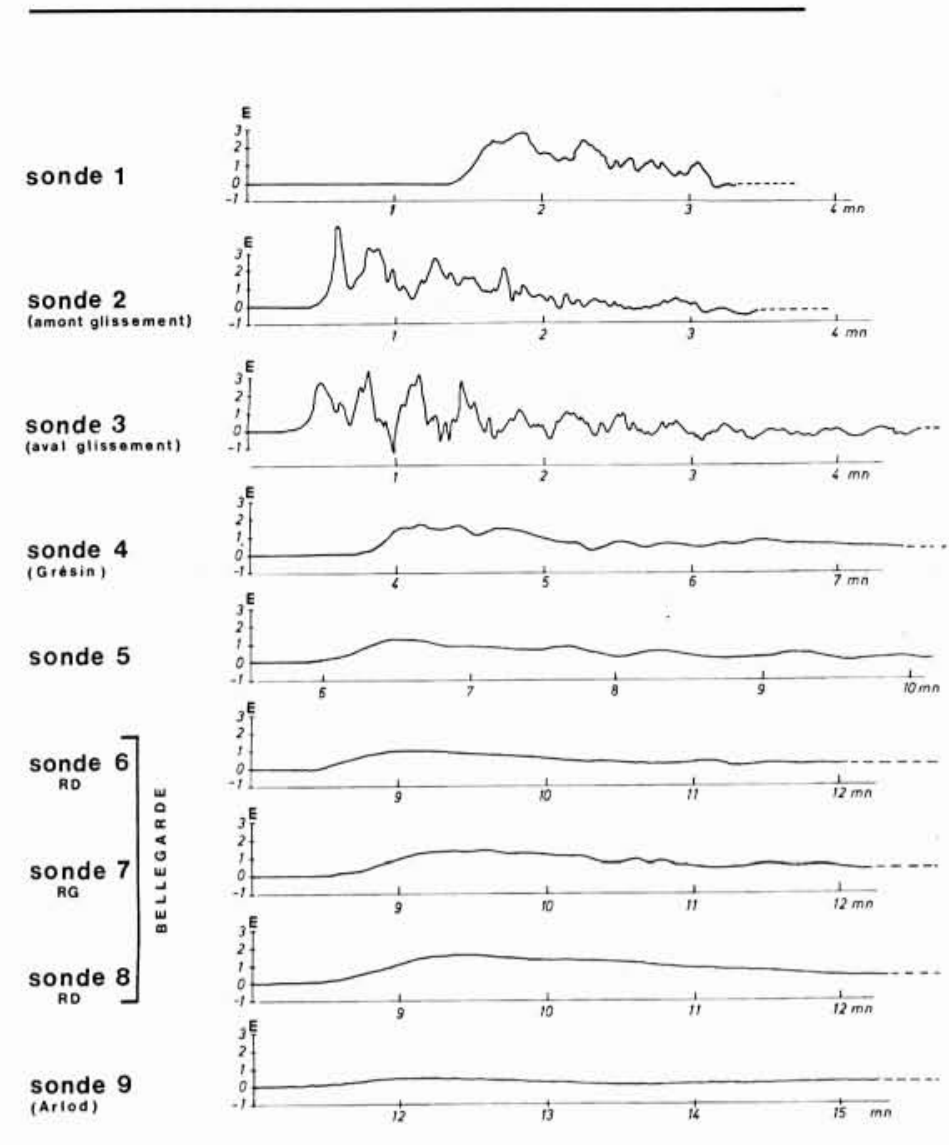

10. Glissement de Léaz - Essai de base.

Evolution des niveaux en différents points de la retenue.

La figure 1la (page suiv.) correspond à l'essai de base réalisé avec des blocs de rhodorsil et représente les ondes mesurées à l'aval du glissement et au pont de Grésin où apparaît le regroupement des différents pics. La figure $11 \mathrm{~b}$ donne à titre d'exemple les enregistrements obtenus en ces deux points, mais avec du sable.

Des essais ont également été effectués en faisant varier la densité du glissement de 1 à 3 afin d'apprécier l'influence du phénomène de décélération plus ou moins important, dû à l'effet de la poussée d'Archimède sur la génération de l'onde. Ces essais effectués avec un volume de glissement de $100000 \mathrm{~m}^{3}$, montrent que l'effet de choc augmente avec la densité ainsi que l'onde dans la retenue, au voisinage du glissement, (l'onde initiale est formée, pour $\mathrm{d}=1,8$ et 3 , de deux pics principaux et, pour $\mathrm{d}=1$, de trois pics). Du fait de la composition différente du train d'ondes, à Bellegarde l'amplitude de l'onde décroît légèrement avec la densité (de $1,05 \mathrm{~m}$ pour $\mathrm{d}=1$ à $0,85 \mathrm{~m}$ pour $\mathrm{d}=3$ ).

Enfin des essais ont été effectués en faisant varier la forme du glissement, en plaçant les différents éléments du glissement côte à côte ou superposés. Les résultats montrent que dans la mesure où le volume immergé est le même, l'onde résultante à Bellegarde est peu modifiée. 


\subsubsection{Influence du débit du Rhône}

Les essais montrent que lorsque le débit du Rhône augmente :

- l'amplitude de l'onde à Bellegarde diminue $(1,40 \mathrm{~m}$ pour $0 \mathrm{~m}^{3} / \mathrm{s}$ à $1,25 \mathrm{~m}$ pour $1300 \mathrm{~m}^{3} / \mathrm{s}$ );

- la vitesse de propagation de l'onde aval augmente (à la célérité de l'onde s'ajoute la vitesse d'écoulement), ce qui accroît les pertes de charges d'où l'amplitude plus faible à Bellegarde.

\subsubsection{Influence de la vitesse du glissement}

La vitesse du glissement probable est de $26 \mathrm{~m} / \mathrm{s}$ et compte tenu du site, ne semble pas pouvoir être dépassée. Par contre, des vitesses plus faibles peuvent être envisagées.

Les essais ont donc porté sur des vitesses comprises entre 6 et $26 \mathrm{~m} / \mathrm{s}$. Il apparaît que lorsque la vitesse augmente, l'amplitude des ondes diminue au voisinage immédiat du glissement. Les différents pics qui composent le train d'ondes, ont des hauteurs variables, et dans un ordre différent suivant la vitesse. La quantité d'eau projetée sur la rive opposée, responsable du deuxième pic et des suivants, augmente avec la vitesse du glissement, tandis que la quantité d'eau chassée directement correspondant au premier pic, diminue.

Lors de leur déplacement, les différents pics se regroupent et on obtient à Bellegarde, une onde d'amplitude pratiquement indépendante de la vitesse du glissement dans l'intervalle de mesure.

La vitesse du glissement n'est pas dans le cas de Léaz un facteur aggravant. En effet ce glissement ne peut communiquer transversalement sa quantité de mouvement à l'écoulement, du fait de l'étroitesse de la retenue et de son orientation perpendiculaire au glissement.

Les figures $11 a$ et $11 c$ font apparaître l'influence sur l'onde de la vitesse du glissement.

\subsubsection{Influence du niveau du plan d'eau}

Lorsque le niveau de la retenue baisse de 330,70 NGF à 325,70 NGF et 323,70 NGF, l'amplitude de l'onde, immédiatement à l'amont et à l'aval du glissement diminue. Le volume d'eau chassé est moindre, d'une part parce que le volume d'eau, au droit du glissement est réduit, et d'autre part, parce que les blocs malgré leur souplesse, n'occupent ảlors qu'imparfaitement la retenue.

La comparaison des figures $11 a$ et $11 d$ permet d'apprécier l'influence du niveau de la retenue aux deux points de mesures.

A Bellegarde l'onde passe de 1,40 à $1,10 \mathrm{~m}$ lorsque la retenue est abaissée de $5 \mathrm{~m}$.

Par sécurité l'amplitude prise en compte pour la définition de la consigne de sécurité appliquée à la retenue, sera celle de la retenue normale.

\subsubsection{Influence de la concomitance du glissement}

Les essais précédents ont été effectués avec un glissement général perpendiculaire à l'axe du Rhône. Des essais ont été effectués pour apprécier l'influence de la non- concomitance du glissement correspondant, par exemple, au cas où la partie aval glisse juste après la partie amont.

On constate que l'onde initiale est renforcée par ce glissement (effet piston): elle atteint notamment $3,9 \mathrm{~m}$ pour les deux premiers pics, contre 2,70 et $3,40 \mathrm{~m}$ dans l'essai de base, avec des creux intermédiaires plus accentués, significatifs du caractère dynamique de cette onde ; la masse d'eau projetée sur la rive opposée est plus faible.

Au pont de Grésin l'augmentation de l'onde est encore de $0,5 \mathrm{~m}$. A Bellegarde, elle n'est que de $0,10 \mathrm{~m}$.

Les figures $11 a$ et $11 e$ montrent l'incidence de ce paramètre sur l'onde.

\subsubsection{Influence du volume du glissement}

Les essais ont été effectués en faisant varier le volume du glissement de 100000 à $1000000 \mathrm{~m}^{3}$. Le glissement était représenté par des blocs de rhodorsil jusqu'à $350000 \mathrm{~m}^{3}$, et par des sacs plastiques remplis de sable et d'eau, pour les volumes supérieurs ou égaux. Au voisinage du glissement, le train d'onde comporte des pics qui augmentent en nombre et en amplitude, en fonction du volume.

A l'aval immédiat du glissement, le maximum atteint :

\begin{tabular}{l|l|r|c}
$1,60 \mathrm{~m}$ & pour & $100000 \mathrm{~m}^{3}$ & avec blocs de \\
$3,40 \mathrm{~m}$ & pour & $350000 \mathrm{~m}^{3}$ & $\begin{array}{r}\text { rhodorsil } \\
3,80 \mathrm{~m}\end{array}$ \\
$6,10 \mathrm{~m}$ & pour & $350000 \mathrm{~m}^{3}$ & avec sacs de \\
$7,30 \mathrm{~m}$ & pour & $500000 \mathrm{~m}^{3}$ & sable
\end{tabular}

A Bellegarde, l'amplitude de l'onde augmente approximativement proportionnellement au volume jusqu'à $500000 \mathrm{~m}^{3}$ environ, puis de plus en plus lentement, et le maximum atteint :

\begin{tabular}{l|l|r|c}
$0,6 \mathrm{~m}$ & pour & $100000 \mathrm{~m}^{3}$ & avec blocs de \\
$1,4 \mathrm{~m}$ & pour & $350000 \mathrm{~m}^{3}$ & $\begin{array}{r}\text { rhodorsil } \\
1,5 \mathrm{~m}\end{array}$ \\
$2,2 \mathrm{~m}$ & pour & $350000 \mathrm{~m}^{3}$ & avec sacs de \\
$2,3 \mathrm{~m}$ & pour & $500000 \mathrm{~m}^{3}$ & sable
\end{tabular}

Les enregistrements de la figure 12 présentent l'évolution de l'onde à l'aval du glissement de Léaz et au pont de Grésin, pour différents volumes du glissement simulés avec le rhodorsil. La figure 13 donne l'amplitude du maximum de l'onde aval en trois points de la retenue, en fonction du volume du glissement.

\subsubsection{Influence de l'orientation du glissement}

Les essais précédents montrent que la génération de l'onde est très particulière du fait de la topographie du site : le glissement provoque la projection d'une masse d'eau importante sur la rive opposée mais transmet assez peu sa quantité de mouvement vers l'amont et l'aval.

Pour étayer ces résultats, un essai fictif a été effectué avec un bloc de rhodorsil correspondant à $100000 \mathrm{~m}^{3}$, lâché parallèlement à l'axe du Rhône et comparé à l'essai normal de même volume, mais se déplaçant perpendiculairement au Rhône. 


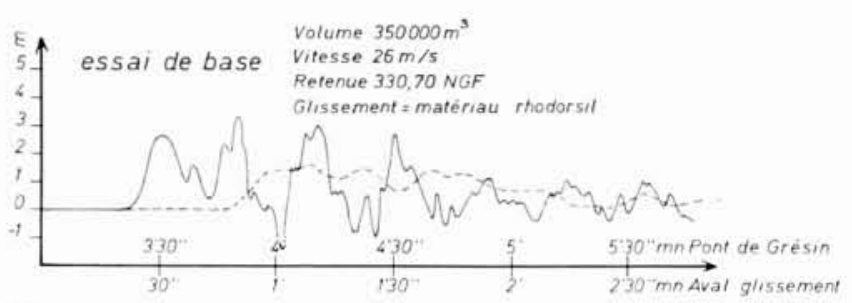

influence de la modélisation (matériau = sable)

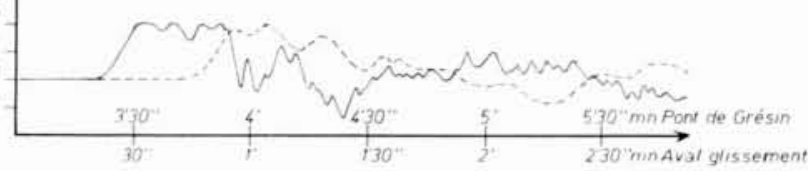

influence de la vitesse $(6 \mathrm{~m} / \mathrm{s}$ )

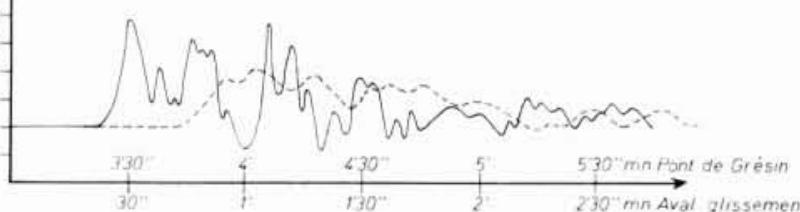

influence du niveau du plan d'eau (323,20 NGF)
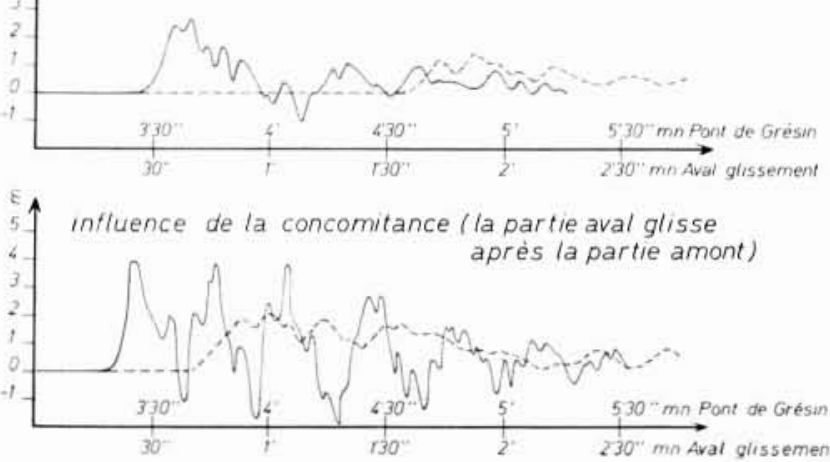

11. Glissement de Léaz.

- Etude de l'influence de divers paramètres.

- Evolution des niveaux à l'aval du glissement PK 24.11 et au pont de Grésin PK 27.10 ....
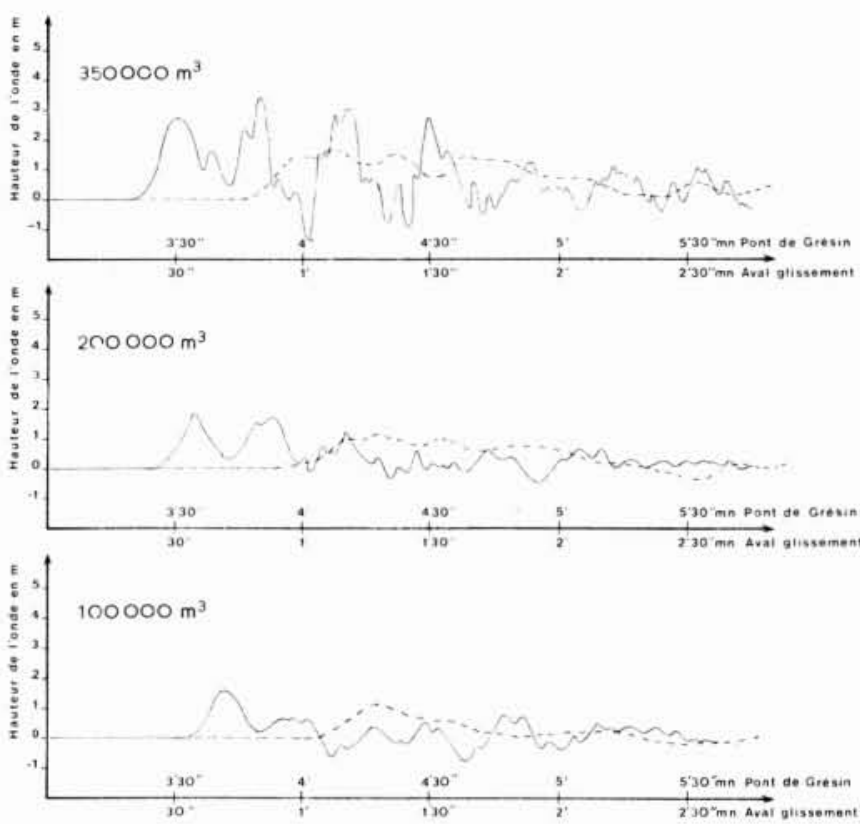

12. Glissement de Léaz.

- Etude de l'influence du volume du glissement.

- Evolution des niveaux à l'aval du glissement pont de Grésin .....

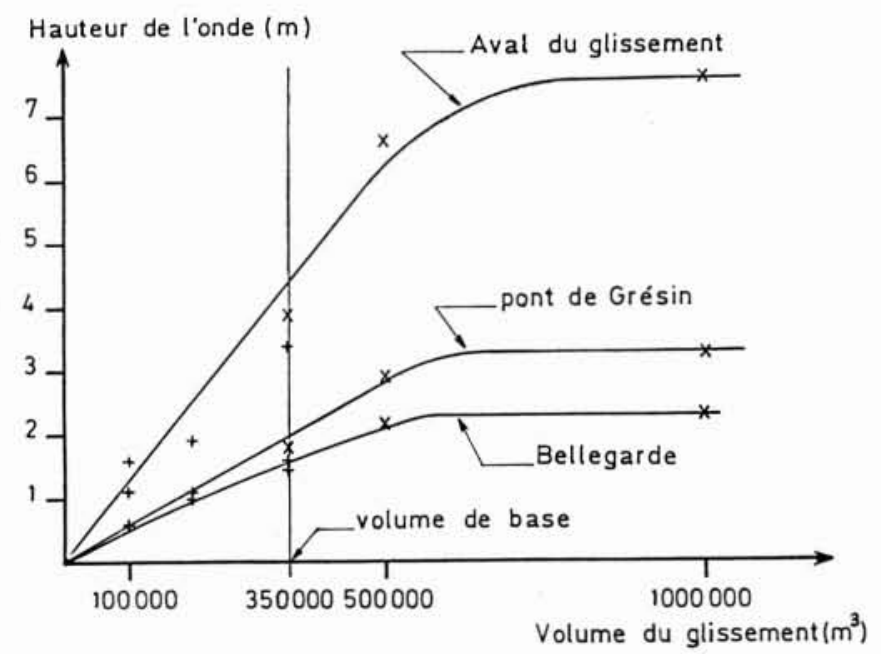

+ glissement monolithique rhodorsil $d=1,8$

$x$ glissement monolithique sacs de sable $d=1,8$

13. Glissement de Léaz.

Hauteur de l'onde en fonction du volume du glissement. 
14. Evaluation de l'amplitude de l'onde par effet piston. a) Profil en travers.

b) Vue en plan.
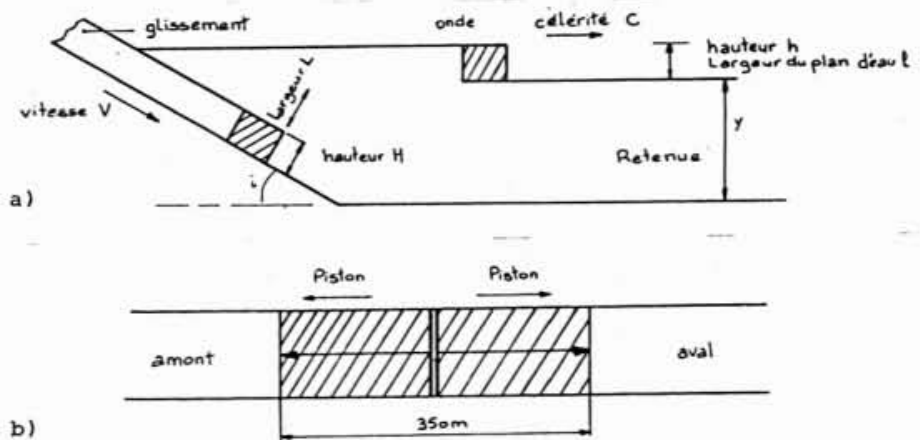

15. Evaluation de l'amplitude de l'onde à partir des volumes mis en jeu.

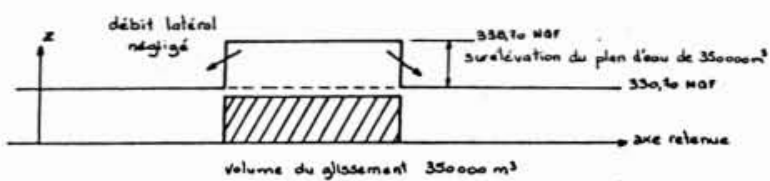

Cet essai fictif conduit à une onde beaucoup plus forte à l'aval immédiat du glissement ( $10,4 \mathrm{~m}$ contre $1,60 \mathrm{~m}$ dans le cas normal), et de forme monochromatique.

L'onde atteint $2,9 \mathrm{~m}$ à Bellegarde contre $0,6 \mathrm{~m}$ dans le cas normal. Cet essai fictif confirme bien la génération particulière de l'onde dans le cas du glissement de Léaz.

\subsection{Approche numérique de l'amplitude de l'onde}

Ces différents résultats permettent également d'apprécier l'adaptation de certaines modélisations mathématiques pour estimer l'amplitude de l'onde.

Par exemple, la modélisation consistant à admettre que le débit du glissement qui entre dans la retenue est égal au débit total des ondes générées vers l'amont et vers l'aval par effet piston, n'est pas applicable au glissement de Léaz (fig. 14a et 14b). En effet ces calculs montrent que pour ce glissement, l'amplitude serait de $17 \mathrm{~m}$ à son voisinage alors que l'on trouve de 4 à $5 \mathrm{~m}$ sur le modèle.

Ces calculs sont seulement valables dans le cas du glissement fictif testé précédemment (ils donnent $12 \mathrm{~m}$ contre $10,4 \mathrm{~m}$ mesuré sur le modèle).

Par contre il est plus vraisemblable d'admettre dans le cas de Léaz, que l'onde au droit du glissement atteint la hauteur de la retenue correspondant à l'augmentation de volume dû au glissement (fig. 15), ce qui conduit à une estimation de la hauteur d'onde un peu supérieure à celle calculée.

Il convient donc d'analyser précisément le phénomène, avant d'opter pour l'une ou l'autre de ces modélisations mathématiques, du fait qu'elles conduisent à des résultats très différents.
De plus ces modélisations ne permettent pas de préciser la forme de l'onde alors qu'elle est déterminante, avec évidemment la topographie de la retenue pour l'évaluation de son écrêtement au cours de sa propagation.

C'est d'ailleurs ce type d'approche simplifié qui avait fait craindre des ondes importantes à Bellegarde-sur-Valserine.

\subsection{Rupture du barrage formé par le glissement}

Le comblement de la retenue au droit du glissement, peut créer un bouchon, susceptible lors de sa rupture, de provoquer une nouvelle onde. Dans le cas où la retenue est à sa cote normale $330,70 \mathrm{NGF}$, le remblai correspondant au glissement normal de $350000 \mathrm{~m}^{3}$, supposé uniformément réparti, serait arasé à $328,00 \mathrm{NGF}$. Il n'y a donc pas de bouchon véritable mais seulement rétrécissement local de la section d'écoulement : le remblai s'érodera progressivement au fur et à mesure du passage des écoulements. Dans le cas où le plan d'eau est à $325,70 \mathrm{NGF}$, le bouchon dépasse de 2,3 m environ. Sa rupture instantanée, d'ailleurs difficilement imaginable, créera une onde initiale estimée à $1,70 \mathrm{~m}$. Cette onde est donc sans danger pour l'aval, puisque le niveau est $5 \mathrm{~m}$ plus bas que la retenue normale.

On a par contre imaginé le cas où le glissement de $350000 \mathrm{~m}^{3}$ n'est pas uniformément réparti, et conduit à un bouchon plus élevé.

En supposant sa crête large de $160 \mathrm{~m}$ à $335,70 \mathrm{NGF}$ (hypothèse très défavorable), et le plan d'eau de la retenue à $325,70 \mathrm{NGF}$, un essai sur modèle a montré que l'érosion du bouchon est progressive et que l'onde atteint un maximum de $1 \mathrm{~m}$ à l'aval du glissement, et de $0,6 \mathrm{~m}$ à Bellegarde. 


\section{Etude du glissement de Grésin}

Profitant de l'existence du modèle et de l'expérience acquise pendant l'étude du glissement de Léaz, le glissement de Grésin a fait l'objet d'essais destinés à tester l'influence de la modélisation (forme et type de matériau), de la vitesse et du volume du glissement (ce volume est mal connu et se situe entre 40000 et $200000 \mathrm{~m}^{3}$ selon les hypothèses d'épaisseur et de surface mises en jeu).

La forme initiale du glissement et celle de la retenue étant différentes (la largeur du glissement varie de 100 à $150 \mathrm{~m}$ suivant les hypothèses, et celle de la retenue est égale à $80 \mathrm{~m}$ ), des essais ont été effectués, en simulant différentes formes du glissement, qui ont donné des résultats voisins ; l'amplitude maximum étant cependant obtenue pour le volume d'eau déplacé maximum.
La génération de l'onde est comparable au glissement de Léaz: submersion de la rive opposée jusqu'à $380 \mathrm{NGF}$, trains d'ondes complexes mais plus courts, se déplaçant de part et d'autre du glissement.

L'onde résultante aval subit un écrêtement sensible au cours de son déplacement mais la proximité de la ville de Bellegarde est un élément défavorable.

Elle y atteint une hauteur de $1,5 \mathrm{~m}$ pour le volume maximum de $200000 \mathrm{~m}^{3}$ hauteur équivalente à celle du glissement de Léaz normal; mais seulement $1 \mathrm{~m}$ pour $100000 \mathrm{~m}^{3}$ et $0,6 \mathrm{~m}$ pour $40000 \mathrm{~m}^{3}$.

Une reconnaissance complémentaire du glissement de Grésin, permettra de préciser le volume mobilisable et de fixer les dispositions à prendre.

\section{Conclusion}

L'étude sur modèle physique au 1/200 des glissements de Léaz et de Grésin, dans la retenue de Génissiat, sur le Rhône, a permis de préciser l'influence de la simulation du glissement et des différents paramètres liés au glissement, et d'en déduire l'onde résultante maximum au droit des zones sensibles, et plus particulièrement de Bellegardesur-Valserine, en fonction du volume du glissement.

Les situations respectives des glissements et des zones sensibles, et les caractéristiques de la retenue de Génissiat, tendent à réduire l'amplitude de l'onde au droit de ces zones.

On observe, en général, un train d'ondes complexe au voisinage du glissement, formé de plusieurs pics. Ces pics sont rapidement amortis au cours de leur déplacement : à Bellegarde-sur-Valserine on observe une onde unique, d'amplitude relativement modeste.
L'amplitude des ondes ne dépend pratiquement que du volume d'eau déplacé et peu des autres paramètres, en particulier de la vitesse du glissement. Ceci est dû à la forme très particulière de la retenue au droit du glissement. L'effet de choc du glissement sur la masse d'eau se transmet sur la rive opposée et très peu à l'amont et à l'aval, conduisant à des amplitudes relativement faibles.

L'effet sur les riverains peut être annihilé grâce à un abaissement préventif de la retenue, réalisé en période d'activité du glissement. Pour le glissement de Léaz normal $\left(350000 \mathrm{~m}^{3}\right)$, un abaissement préventif du plan d'eau de $2 \mathrm{~m}$ minimum au-dessous de la retenue normale (contre $5 \mathrm{~m}$ actuellement), assure une sécurité suffisante.

L'étude a montré la complexité de la génération des trains d'ondes, typiquement tridimensionnelles, et le caractère indispensable du modèle réduit.

\section{Study on a reduced model of the Léaz slippage in the Génissiat barrage}

A potential landslide of approximately $350,000 \mathrm{~m}^{3}$ is located on the right bank of the Rhone, above the barrage created by the Génissiat Dam, approximately 7 kilometers upstream from the built-up area of Bellegarde-sur-Valserine. This has led the Compagnie Nationale du Rhone to constantly supervise the area and to carry out various studies intended to draw up a set of urgent operating procedures in order to avoid any damage for the inhabitants. The aim of the reducedmodel study described here is to determine the consequences of a landslide on the Génissiat barrage and on the town of Bellegarde-sur-Valserine and thus enable operating procedures to be clearly defined. 